\title{
Lipids and Fatty Acids in Artemia Nauplii Enriched with Fish Oil Triacylglycerols Containing Docosahexaenoic Acid in Different Positional Distribution Patterns
}

\author{
Yasuhiro Ando, Mari Kotake, and Toru Ota \\ Department of Marine Bioresources Chemistry, Faculty of Fisheries, Hokkaido University, \\ Minato, Hakodate 041, Japan
}

(Received August 9, 1996)

\begin{abstract}
Lipid profiles were compared between Artemia nauplii enriched with fish oil triacylglycerols (TG) containing docosahexaenoic acid (DHA, 22:6n-3) in different positional distribution patterns. Four fish oils were selected on the basis of the positional distribution determined by a nonenzymatic analytical method, and directly given to Artemia nauplii in the form of oil emulsions. Time-courses of the lipid contents, lipid compositions, and fatty acid compositions were not so different between the nauplii enriched with tuna orbital and bonito head oil TG, in which 22:6n-3 (22.9-24.0\%) was esterified in the $s n$ $3>s n-1 \gg s n-2$ positions and the $s n-3 \gg s n-2=s n-1$ positions, respectively. In both of the enrichments, the level of $22: 6 \mathrm{n}-3$ initially not detected reached a plateau (3.4-3.6\% of total fatty acids) after $12 \mathrm{~h}$ and then decreased to $1.7 \%$ ( $48 \mathrm{~h}$ ). Slight differences were also observed between the lipids of nauplii fed on sardine $(22: 6 n-3,11.5 \% ; s n-2 \gg s n-3>s n-1)$ and seal $(22: 6 n-3,7.6 \% ; s n-3>s n-1>s n-2)$ oil TG. The levels of 22:6n-3 after $24 \mathrm{~h}$ enrichment were $0.4 \%$ of total fatty acids. Such similarities of the lipid profiles in Artemia nauplii indicate that the positional distribution of 22:6n-3 in dietary TG has practically no influence to enrichment of Artemia nauplii with this fatty acid. It is probable that any TG with different positional distribution patterns of $22: 6 n-3$ can be used for enrichment of Artemia, when $22: 6 n-3$ is concentrated in the TG at an adequate level.
\end{abstract}

Key words: Artemia, fish oil, triacylglycerol, fatty acid, docosahexaenoic acid, n-3 HUFA, positional distribution, enrichment

Longer-chain n-3 highly unsaturated fatty acids (n-3 HUFA) such as eicosapentaenoic acid (EPA; 20:5n-3) and docosahexaenoic acid (DHA; 22:6n-3) are essential dietary components for marine fish. " Of these fatty acids, 22:6n-3 has been shown to be a more efficient essesntial fatty acid for some fish larvae and juveniles. ${ }^{2)}$ Artemia nauplii are used in aquaculture as an importent live food for fish larvae. As many strains of Artemia can not provide an adequate amount of n-3 HUFA, ${ }^{3-5)}$ the nauplii are usually enriched with a diet rich in $\mathrm{n}-3$ HUFA prior to being fed to fish larvae. A number of different enrichment diets have been successfully used including micro-algae, n-3 HUFAmodified yeasts, compound diets, coated microparticles, oil-based emulsions, and microencapsulated preparations. ${ }^{6,7)}$ In the enrichment diets, n-3 HUFA are present in some forms such as triacylglycerols (TG), ethyl esters, methyl esters, glyceroglycolipids, and glycerophospholipids. Takeuchi et al. ${ }^{8)}$ compared the incorporation of n-3 HUFA in Artemia among the types of TG, ethyl esters, methyl esters, and free fatty acids using the oil-based emulsions. Although the maximal incorporation of n-3 HUFA was found in enrichment with ethyl ester-type oil, comparable incorporation is observed in their experimental data on TG-type oil.

Influence of positional distribution of n-3 HUFA to enrichment diet $\mathrm{TG}$ is one of the interesting subjects. When TG are subjected to pancreatic lipase hydrolysis, ester bonds of $20: 5 n-3$ and $22: 6 n-3$ in the $s n-1(3)$ position are hydrolyzed more slowly than those of other fatty acids. ${ }^{9}$ ) These fatty acids were mainly found in 1,2(2,3)-diacylglycerols and TG of the hydrolysis products, suggesting that they are resistant to the action of lipase. ${ }^{10)}$ Such findings lead us to associate the positional distribution of $n-3$ HUFA in dietary TG with an effective enrichment of Artemia with them. There has been no report on influence of the positional distribution, because the ordinary enzymatic method for determination of positional distribution (i.e., stereospecific analysis of TG) is unsuitable for the application to n-3 HUFA-containing TG such as marine oils. ${ }^{11)}$

In the present study, four fish oils (tuna orbital, bonito head, sardine, and seal oils) have been selected and given to Artenia nauplii as emulsified oils. Lipid contents, lipid compositions, and fatty acid compositions were compared between the nauplii enriched with the fish oil TG containing 22:6n-3 in different positional distribution patterns. Positional ditributions of fatty acids in the fish oil TG used had been determined by a nonenzymatic analysis and reported in our previous papers. ${ }^{12,13)}$

\section{Materials and Methods}

\section{Fish Oil TG Used for Enrichment}

Tuna orbital, bonito head, sardine, and seal oils were used for enrichment. All of the oils were industrial products. Positional distributions of fatty acids in TG of 
these fish oils had been detailed in our previous reports. ${ }^{12,13)}$ Nonenzymatic analysis of them was in short carried out by way of preparation of 1(3)- and 2-monoacylsn-glycerols (MG) by Grignard hydrolysis of TG, separation of 1- and 3-MG by chiral high-performance liquid chromatography after derivatization, and fatty acid analysis of the TG and MG by gas-liquid chromatography (GLC). Tables 1 and 2 show the analytical data on major fatty acids. The proportions of $22: 6 \mathrm{n}-3$ in total fatty acids of TG were 22.9 and 24.0 mole \% in the tuna orbital and bonito head oils, respectively. In the tuna orbital oil TG, $22: 6 n-3$ was preferentially esterified in the $s n-3$ position (33.2 mole \%), followed by the $s n-1$ position ( $24.7 \mathrm{~mole} \%$ ). The content of this acid in the $s n-2$ position was 5.5

Table 1. Positional ditribution of fatty acids in triacylglycerols of tuna orbital and bonito head oils (mole $\%)^{*}$

\begin{tabular}{|c|c|c|c|c|c|c|c|c|}
\hline \multirow{2}{*}{ Fatty acid } & \multicolumn{4}{|c|}{ Tuna orbital oil ${ }^{13)}$} & \multicolumn{4}{|c|}{ Bonito head oill3\} } \\
\hline & Total & $s n-1$ & $s n-2$ & $s n-3$ & Total & $s n-1$ & $s n-2$ & $s n-3$ \\
\hline $14: 0$ & 4.2 & 2.8 & 9.0 & 2.2 & 4.7 & 3.5 & 8.5 & 2.5 \\
\hline $15: 0$ & 1.1 & 1.0 & 1.9 & 0.6 & 1.1 & 0.9 & 1.7 & 0.7 \\
\hline $16: 0$ & 22.1 & 24.5 & 31.5 & 13.5 & 20.7 & 31.0 & 22.4 & 10.4 \\
\hline $16: 1 n-7$ & 6.3 & 4.7 & 9.9 & 5.1 & 7.1 & 8.5 & 8.9 & 4.5 \\
\hline $16: 2 n-4$ & 1.9 & 0.6 & 3.4 & 2.0 & 1.7 & 1.0 & 2.8 & 1.5 \\
\hline $17: 0$ & 1.0 & 1.3 & 1.4 & 0.6 & 1.2 & 1.5 & 1.5 & 0.6 \\
\hline $18: 0$ & 4.5 & 7.1 & 2.8 & 3.4 & 4.1 & 0.2 & 0.9 & 0.1 \\
\hline $18: \ln -9$ & 15.2 & 15.4 & 12.5 & 16.8 & 13.2 & 16.8 & 10.1 & 14.7 \\
\hline $18: \ln -7$ & 2.6 & 2.9 & 2.5 & 2.5 & 2.6 & 3.9 & 2.2 & 2.0 \\
\hline $18: 2 n-6$ & 2.2 & 1.7 & 3.3 & 2.0 & 1.5 & 1.4 & 1.7 & 1.4 \\
\hline $18: 4 n-3$ & 0.9 & 0.5 & 1.7 & 0.7 & 1.0 & 0.7 & 1.7 & 0.8 \\
\hline $20: \ln -9$ & 1.0 & 0.9 & 0.5 & 1.4 & 0.9 & 0.9 & 0.7 & 1.5 \\
\hline $20: 4 n-6$ & 1.6 & 1.6 & 2.3 & 1.1 & 1.9 & 1.0 & 2.3 & 2.8 \\
\hline $20: 5 n-3$ & 5.8 & 4.0 & 6.4 & 7.1 & 7.1 & 5.0 & 8.2 & 8.1 \\
\hline $22 \div 5 n-6$ & 1.4 & 1.0 & 0.7 & 2.1 & 1.5 & 0.6 & 0.7 & 3.3 \\
\hline $22: 5 n-3$ & 1.1 & 1.0 & 1.1 & 1.1 & 1.3 & 0.7 & 2.2 & 1.1 \\
\hline $22: 6 n-3$ & 22.9 & 24.7 & 5.5 & 33.2 & 24.0 & 18.3 & 19.6 & 37.6 \\
\hline Others $(<1 \%)$ & 4.2 & 4.3 & 3.6 & 4.6 & 4.4 & 4.1 & 3.9 & 6.4 \\
\hline
\end{tabular}

* Values are means for duplicate analyses.

Table 2. Positional ditribution of fatty acids in triacylglycerols of sardine and seal oils (mole\%) ${ }^{*}$

\begin{tabular}{|c|c|c|c|c|c|c|c|c|}
\hline \multirow{2}{*}{ Fatty acid } & \multicolumn{4}{|c|}{ Sardine oil12) } & \multicolumn{4}{|c|}{ Seal oil ${ }^{13)}$} \\
\hline & Total & $s n-1$ & $s n-2$ & $s n-3$ & Total & $s n-1$ & $s n-2$ & $s n-3$ \\
\hline $14: 0$ & 8.9 & 9.6 & 8.6 & 8.4 & 6.3 & 3.3 & 14.8 & 3.8 \\
\hline $16: 0$ & 20.5 & 25.5 & 16.8 & 19.4 & 8.1 & 9.1 & 10.0 & 5.6 \\
\hline $16: 1 \mathrm{n}-7$ & 5.6 & 7.2 & 4.8 & 4.9 & 22.8 & 17.5 & 33.6 & 19.4 \\
\hline $16: 2 n-4$ & 0.6 & 0.6 & 0.6 & 0.7 & 1.2 & 0.7 & 2.0 & 1.1 \\
\hline $18: 0$ & 2.5 & 4.0 & 1.1 & 2.5 & 0.9 & 0.8 & 1.0 & 0.8 \\
\hline $18: \ln -9$ & 9.4 & 11.1 & 6.3 & 10.7 & 17.8 & 18.2 & 20.0 & 15.5 \\
\hline $18: 1 \mathrm{n}-7$ & 2.9 & 4.3 & 1.5 & 2.9 & 5.6 & 6.9 & 4.3 & 5.2 \\
\hline $18: 2 n-6$ & 1.6 & 2.0 & 1.2 & 1.6 & 1.5 & 1.3 & 3.0 & 0.7 \\
\hline $18: 3 n-3$ & 1.2 & 1.4 & 0.7 & 1.3 & 0.5 & 0.5 & 0.6 & 0.3 \\
\hline $18: 4 n-3$ & 3.8 & 4.1 & 3.8 & 3.6 & 1.2 & 1.4 & 0.4 & 1.5 \\
\hline $20: 1 \mathrm{n}-11,13$ & 2.8 & 2.5 & 2.3 & 3.7 & 1.2 & 1.8 & 0.5 & 1.3 \\
\hline $20: \ln -9$ & 1.7 & 1.8 & 1.4 & 1.8 & 6.7 & 11.5 & 1.4 & 5.9 \\
\hline $20: 5 n-3$ & 11.7 & 9.0 & 12.2 & 13.7 & 8.0 & 7.3 & 1.4 & 13.4 \\
\hline $22: \ln -11,13$ & 3.0 & 2.0 & 2.8 & 4.2 & 2.2 & 4.9 & 0.3 & 1.0 \\
\hline $22: 5 n-3$ & 1.5 & 0.8 & 2.7 & 1.1 & 4.5 & 3.0 & 0.6 & 8.7 \\
\hline $22: 6 n-3$ & 11.5 & 3.1 & 22.1 & 9.1 & 7.6 & 7.2 & 1.4 & 12.5 \\
\hline Others $(<1 \%)$ & 10.7 & 10.9 & 11.2 & 10.4 & 3.9 & 4.6 & 4.7 & 3.3 \\
\hline
\end{tabular}

* Values are means for duplicate analyses. mole $\%$. The bonito head oil TG contained $22: 6 \mathrm{n}-3$ at the concentrations of $18.3,19.6$, and 37.6 mole \% in the $s n-1$ $s n-2$, and $s n-3$ positions, respectively. In this fish oil, $22: 6 n-3$ was primarily located in the $s n-3$ position, and distributed almost evenly between the $s n-1$ and $s n-2$ positions. The contents of $22: 6 \mathrm{n}-3$ in the sardine (11.5 mole $\%)$ and seal oils ( 7.6 mole $\%)$ were much lower than those in the tuna orbital and bonito head oils (Table 2). The sardine oil TG contained $22: 6 n-3$ in the $s n-2$ position $(22.1$ mole $\%)$ at the concentration higher than in the $s n-1$ (3.1 mole \%) and $s n-3(9.1$ mole $\%)$ positions. In the seal oil TG, 22:6n-3 was more concentrated in the $s n-3$ position $(12.5 \mathrm{~mole} \%)$, followed in sequence by the $s n-1 \quad(7.2$ mole $\%)$ and $s n-2(1.4 \mathrm{~mole} \%)$ positions. The tuna orbital and bonito head oils were dried over anhydrous sodium sulfate as $n$-hexane solutions, and then used without further purification. The sardine and seal oil TG were isolated by column chromatography on Silica gel 60 (Merck, Darmstadt, Germany) using mixtures of $n$-hexane/diethyl ether as solvents.

\section{Enrichment of Artemia with Oil Emulsion}

Enrichment of Artemia was carried out by the direct method. ${ }^{4,5,8)}$ The Artemia used in this study originated from the Great Salt Lake, Utah, USA (Nisshin Science, Yokohama, Japan). Newly-hatched nauplii of Artemia were stocked into $2 l$ flat-bottom flasks at a density of 100 individuals $/ \mathrm{ml}$ and enriched by adding $1.8 \mathrm{~m} /$ of oil emulsion to the medium at $28^{\circ} \mathrm{C}$. The enrichment was carried out under vigorous aeration. In all the experiments, $90 \mathrm{mg}$ of oil was emulsified with $22.5 \mathrm{mg}$ of egg york lecitin PL100E (Research Institute Q.P., Tokyo, Japan) and $1.8 \mathrm{ml}$ of distilled water using a micro-homogenizer Physcotron NS-310E (Niti-on, Funabashi, Japan) at 18,000 rpm for 2 min. After the oil emusions were poured into the culture medium, Artemia nauplii samples $(200 \mathrm{~m} /$ of the medium) were taken on filter paper at $0,6,12,24$, and $48 \mathrm{~h}$, washed three times with $100 \mathrm{~m} l$ of distilled water, and stored at $-35^{\circ} \mathrm{C}$.

\section{Analyses of Lipids and Fatty Acids}

Lipids were extracted by the method of Bligh and Dyer, ${ }^{14)}$ and the total lipids were determined gravimetrically. Lipid class composition was determined with Iatroscan TH-10 (Iatron Laboratories, Tokyo, Japan) spotting chloroform solutions of the extracts on chromarod SIII and using benzene/chloroform/acetic acid $(50: 20: 1, v / v / v)$ as development solvents. Fatty acids were analyzed by GLC after methylation with a mixture of benzene and sodium methoxide in methanol $(0.5 \mathrm{M})$. Fatty acid methyl esters were purified by thin-layer chromatography on Silica gel $60 \mathrm{G}$ plate (Merck) with $n$-hexane/diethyl ether (90:10, $\mathrm{v} / \mathrm{v}$ ) for development. GLC analyses of the methyl esters were performed on Shimadzu GC-14A and GC-17A gas chromatographs (Shimadzu Co., Kyoto, Japan) equipped with capillary columns Omegawax $320(30 \mathrm{~m} \times 0.32 \mathrm{~mm}$ i.d.; Supelco, Bellefonte, PA, USA) and Omegawax 250 (30 $\mathrm{m} \times 0.25 \mathrm{~mm}$ i.d.; Supelco), respectively, and flame ionization detectors. Column temperatures were 200 (Omegawax 320) and 210 (Omegawax 250) ${ }^{\circ} \mathrm{C}$. Injector and detector temperatures were 250 and $260^{\circ} \mathrm{C}$, respectively. The carrier gases were herium (Omegawax 320) and 
hydrogen (Omegawax 250). Peak area percentages were measured with a Shimadzu Chromatopac C-R6A integrator.

\section{Results and Discussion}

\section{Lipids of Artemia Enriched with Tuna Orbital and Bonito Head Oils}

Figure 1 shows the changes in lipid contents and lipid class compositions of Artemia nauplii enriched with tuna orbital and bonito head oils. The lipid contents based on dry-weight increased about 1.5 times after $12-24 \mathrm{~h}$ of enrichment. The highest values of the contents were not so different between the Artemia enriched with tuna orbital $(32.4 \%)$ and bonito head $(34.0 \%)$ oils. Changes in lipid compositions were also similar between the two enrichments. The levels of $\mathrm{TG}$ and polar lipids (PL) remained almost steady during 0-24 h, and then showed significant decrease and increase, respectively. After $48 \mathrm{~h}$ of enrichment, lipid contents based on wet-weight decreased with the decrease of TG.

Figure 2 shows the proportions of major fatty acids in Artemia nauplii enriched with bonito head and tuna orbital oils. Changes in the proportions of each fatty acid were similar between the Artemia enriched with the two oils. In both the enrichments, concentrations of $22: 6 n-3$ increased with enrichment period, reached maxima after 12 $\mathrm{h}$, and dropped during 12-48 $\mathrm{h}$. The maximal concentrations of $22: 6 n-3$ were 3.6 and $3.4 \%$ in the enrichments with
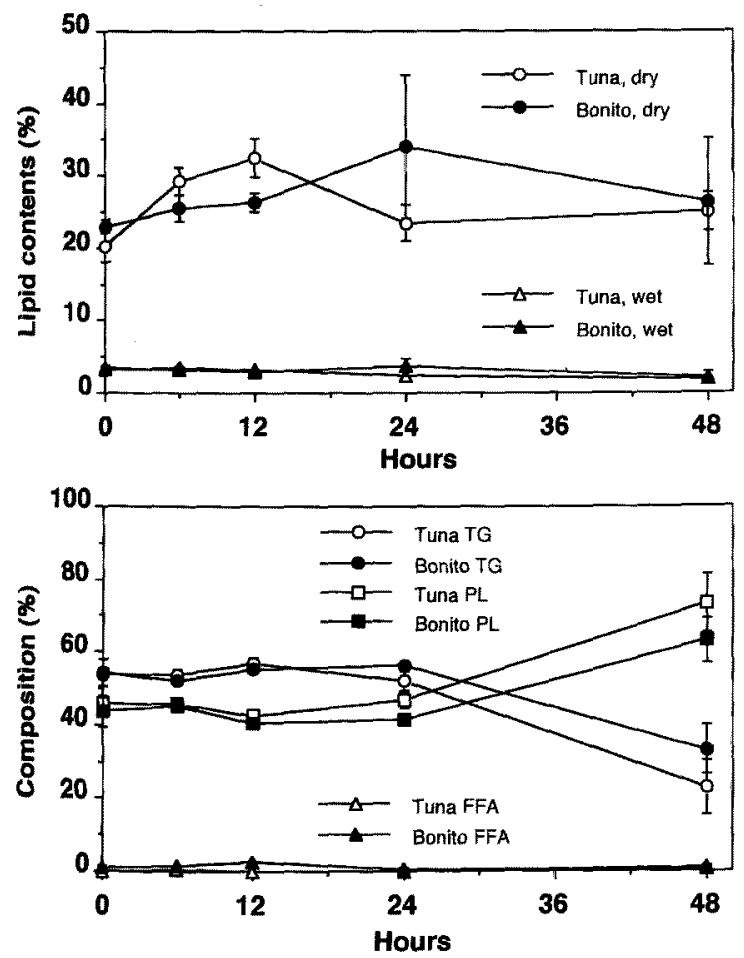

Fig. 1. Lipid contents (dry- and wet-weight bases) and lipid class compositions of Artemia nauplii enriched with tuna orbital and bonito head oils.

Values are means \pm standard deviations for quadruplicate experiments. tuna orbital and bonito head oils, respectively. The levels of $20: 5 n-3$ consistently increased to about $7 \%$ during the enrichment period. Similarly, 20:4n-6 showed consistent increases during the enrichments. The levels of 18:2n-6, $18: 3 n-3$, and $18: 4 n-3$ decreased during the enrichment period.

\section{Lipids of Artemia Enriched with Sardine and Seal Oil TG}

Figure 3 compares the lipid contents and lipid class compositions of Artemia nauplii enriched with sardine and seal oil TG. Lipid contents (wet-weight base) after $24 \mathrm{~h}$ were similar between the two enrichments, and lower than those at initial time. In both the enrichments, proportions of TG and polar lipids showed slight increase and decrease after $24 \mathrm{~h}$ of enrichment, respectively. The levels of these lipid classes were not significantly different between the two enrichments.

Proportions of major fatty acids in Artemia nauplii en-
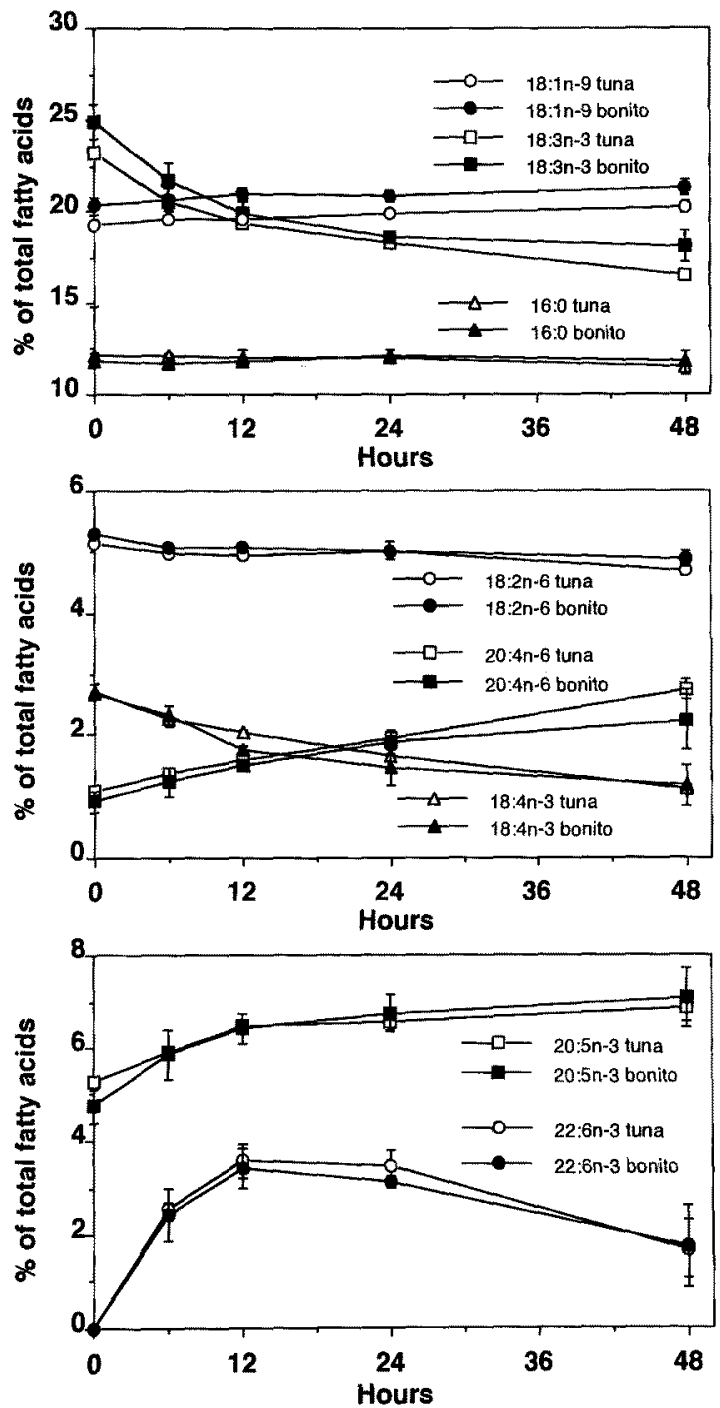

Fig. 2. Fatty acid compositions of Artemia nauplii enriched with tuna orbital and bonito head oils.

Values are means \pm standard deviations for quadruplicate experiments. 

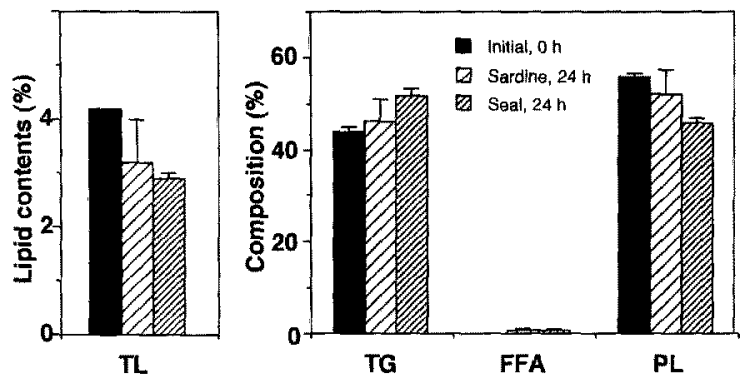

Fig. 3. Lipid contents (wet-weight base) and lipid class compositions of Artemia nauplii enriched with sardine and seal oil triacylglycerols. Values are means \pm standard deviations for triplicate experiments.
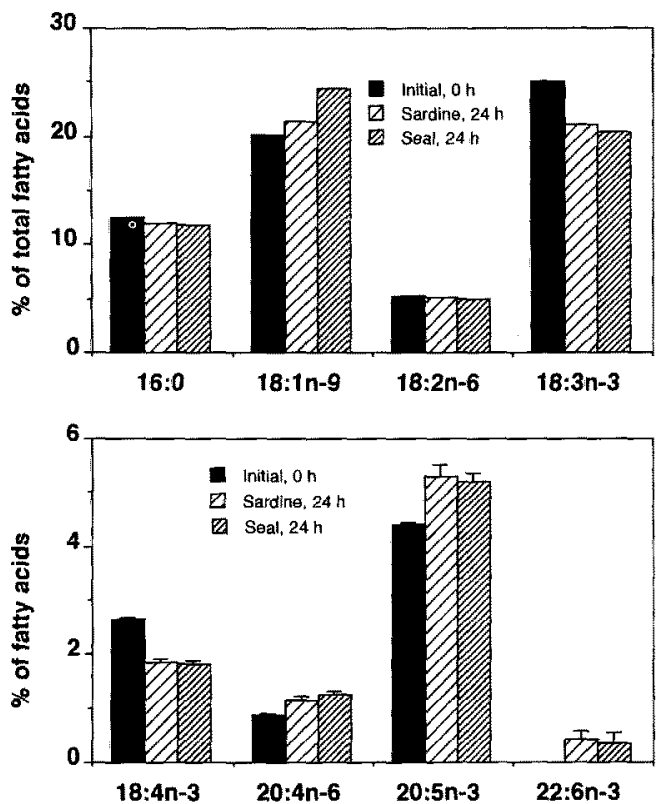

Fig. 4. Fatty acid compositions of Artemia nauplii enriched with sardine and seal oil triacylglycerols.

Values are means \pm standard deviations for triplicate experiments.

riched with sardine and seal oil TG are indicated in Fig. 4. The concentrations of $22: 6 n-3,20: 5 n-3$, and $20: 4 n-6$ increased after $24 \mathrm{~h}$ of enrichment, while 18:3n-3 and 18:4n3 showed decreases. When compared between the two enrichments, there were little differences in proportions of such polyunsaturated fatty acids. The levels of $22: 6 n-3$ in total fatty acids were $0.4 \%$ in both the enrichments. The proportions of $20: 5 n-3,20: 4 n-6,18: 3 n-3$, and $18: 4 n-3$ were $5.2-5.3,1.1-1.3,20.5-21.1$, and $1.8 \%$, respectively.

\section{Influence of Positional Distribution of 22:6n-3 in Fish Oil} $T G$

There were practically no differences in lipid content, lipid composition, and fatty acid composition between $A r$ temia enriched with tuna orbital and bonito head oils. Thus, 22:6n-3 contents in Artemia enriched with the two oils were similar to each other. In the fish oils used for enrichment, positional distributions of $22: 6 n-3$ were different from each other, while the contents of this acid were similar between the two fish oils. Little difference of the 22:6n3 contents was also observed between the Artemia enriched with sardine and seal oil TG. These similarities of the 22:6n-3 contents in Artemia nauplii show that the enrichment with $22: 6 n-3$ is not affected by the positional distribution of this acid in dietary fish oil TG.

In contrast, the 22:6n-3 levels in Artemia enriched with the bonito head and tuna orbital oils ( $24 \mathrm{~h}$ ) were 8-9 times higher as those enriched with the sardine and seal oil TG. The proportions of $22: 6 n-3$ were $22.9-24.0 \%$ in the bonito head and tuna orbital oils and $7.6-11.5 \%$ in the sardine and seal oils TG. High concentration of $22: 6 n-3$ in TG is found to be effective and essential for the enrichment of Artemia with this acid.

\section{Incorporation of 22:6n-3 Esterified in Fish Oil TG}

Takeuchi et al. ${ }^{8)}$ compared the incorporation of lipids and n-3 HUFA in Artemia nauplii among various types of oils, such as TG, ethyl esters, methyl esters, and free fatty acids containing $43 \%$ of n-3 HUFA using the direct method. In their enrichments with ester-type oils, the TG content in Artemia increased and reached a plateau after $24 \mathrm{~h}$. This result suggested that ester oils such as ethyl and methyl esters were very quickly converted to TG and incorporated into TG fraction in Artemia. Similar changes were observed in their enrichment with TG. Rapid hydrolysis and re-constitution of TG seems to occur, when fish oil TG are fed to Artemia nauplii by the direct method. In the present study, changes in contents of 22:6n-3 were similar between Artemia enriched with fish oil TG containing this acid in different positional distribution patterns. This result suggests that the rapid hydrolysis and re-constitution of TG is independent of the positions in dietary TG in which $22: 6 \mathrm{n}-3$ is esterified.

Hayashi et al. ${ }^{(5)}$ suggested the retroconversion of 22:6n3 to 20:5n-3 in Artemia. In their study, a steep down of the 22:6n-3 content in Artemia was observed after 6-12 h of the enrichment, while the content of $20: 5 n-3$ tended to increase in this period. This retoconversion was partially confirmed by Takeuchi et al. ${ }^{*}$ When Artemia were fed on $\left[1-{ }^{14} \mathrm{C}\right]-22: 6 n-3$ together with fish oil ethyl esters, radioactivity was detected in docosapentaenoic acid $(22: 5)$ fraction. In the present study, the 22:6n-3 level in Artemia fed on bonito head and tuna orbital oils dropped in the late period of enrichment, whereas $20: 5 n-3$ increased gradually during the period. This result supports the occurence of the retroconversion in Artemia. Similarity of the changes in 22:6n-3 contents suggests that the process of retroconversion is independent of the position in dietary TGs in which $22: 6 n-3$ is esterified.

Positional distributions of $22: 6 \mathrm{n}-3$ in TG are different among the origins. Sardine, cod liver, and squid liver oils have been frequently used for n-3 HUFA enrichment of live foods such as Artemia and rotifers, ${ }^{4,5,8)}$ In these ordinary fish oils, 22:6n-3 was preferentially esterified in the $s n-2$ position of TG. ${ }^{12,16-18)}$ Marine mammal oils such as seal and whale oils showed the preferential association of 
22:6n-3 to the $s n-3$ position. ${ }^{13,16-18)}$ The distribution pattern in 22:6n-3-rich tuna orbital and bonito head oils was similar to that observed for seal oil rather than sardine oil. ${ }^{\mathrm{I} \text { ) }}$ Moreover, positional distribution of fatty acids can be modified by chemical, enzymatic, or physical treatments such as transesterification and winterization. The present study revealed that the positional distribution of $22: 6 n-3$ in dietary TG has essentially no influence to enrichment of Artemia with this acid. When $22: 6 n-3$ is concentrated in $\mathrm{TG}$ at an adequate level, it is probable that any fish oil TG with different origins can be used for enrichment of Artemia with 22:6n-3.

\section{References}

1) R. G. Ackman and J. K.-Howie: Fatty acids in aquaculture: Are $\omega$ 3 fatty acids always important?, in "Nutrition and Utilization Technology in Aquaculture"' (ed. by C. E. Lin and D. J. Sessa), AOCS Press, Champeign, IL, 1995, pp. 82-104.

2) A. Kanazawa: Nutrition of laeval fish, in "Nutrition and Utilization Technology in Aquaculture" (ed. by C. E. Lin and D. J. Sessa), AOCS Press, Champeign, IL, 1995, pp. 50-59.

3) T. Watanabe, F. Oowa, C. Kitajima, and S. Fujita: Relationship between dietary value of brine shrimp Artemia salina and their content of $\omega 3$ highly unsaturated fatty acids. Nippon Suisan Gakkaishi, 46, 35-41 (1980).

4) T. Watanabe, M. Ota, C. Kitajima, and S. Fujita: Improvement of dietary value of brine shrimp Artemia salina for fish larvae by feeding them on $\omega 3$ highly unsaturated fatty acids. Nippon Suisan Gakkaishi, 48, 1775-1782 (1982).

5) T. Watanabe, T. Tamiya, A. Oka, M. Hirata, C. Kitajima, and S. Fijita: Improvement of dietary value of live foods for fish larvae by feeding them on $\omega 3$ highly unsaturated fatty acids and fat-soluble vitamins. Nippon Suisan Gakkaishi, 49, 471-479 (1983).

6) P.C. Southgate and D.C. Lou: Improving the n-3 HUFA composi- tion of Artemia using microcapsules containing marine oils. Aquaculture, 134, 91-99 (1995).

7) P. Leger, D. A. Bengtson, K. L. Simpson, and P. Sorgeloos: The use and nutritional value of Artemia as a food science. Oceanogr. Mar. Biol. Annu. Rev., 24, 521-623 (1986).

8) T. Takeuchi, M. Toyota, and T. Watanabe: Comparison of lipid and n-3 highly unsaturated fatty acid incorporations between Artemia enriched with various types of oil by direct method. Nippon Suisan Gakkaishi, 58, 277-281 (1992).

9) W. W. Christie: Lipid Analysis, 2nd ed., Pergamon Press, Oxford, 1982, pp. 155-161.

10) N. R. Bottino, G. A. Vandenburg, and R. Reiser: Resistance of certain long-chain polyunsaturated fatty acids of marine oils to pancreatic lipase hydrolysis. Lipids, 2, 487-493 (1967).

11) T. Takagi and Y. Ando: Stereospecific analysis of triacyl-snglycerols by chiral high-performance liquid chromatography. Lipids, 26, 542-547 (1991).

12) Y. Ando, K. Nishimura, N. Aoyanagi, and T. Takagi: Stereospecific analysis of fish oil triacyl-sn-glycerols. J. Am. Oil Chem. Soc., 69, 417-424 (1992).

13) Y. Ando, T. Ota, Y. Matsuhira, and K. Yazawa: Stereospecific analysis of triacyl-sn-glycerols in docosahexaenoic acid-rich fish oils. $J$. Am Oil Chem. Soc., 73, 483-487 (1996).

14) E. G. Bligh and W. J. Dyer: A rapid method of total lipid extraction and purification. Can. J. Biochem. Physiol., 37, 911-917 (1959).

15) M. Hayashi, K. Toda, T. Yoneji, O. Sato, and S. Kitaoka: Dietary value of rotifers and Artemia enriched with Euglena gracilis for red sea bream. Nippon Suisan Gakkaishi, 56, 1051-1058 (1993).

16) H. Brockerhoff, R. J. Hoyle, P. C. Hwang, and C. Litchfield: Positional distribution of fatty acids in depot triglycerides of aquatic animals. Lipids, 3, 24-29 (1968).

17) C. Lithfield: Predicting the positional distribution of docosahexaenoic and docosapentaenoic acids in aquatic animal triglycerides. Lipids, 3, 417-419 (1968).

18) C. Litchfield: Taxonomic patterns in the triglyceride structures of natural fats. Fette Seifen Anstrichm., 75, 223-231 (1971). 